\title{
Research Article \\ Coupled Fixed Point Theorems for Nonlinear Contractions Satisfied Mizoguchi-Takahashi's Condition in Quasiordered Metric Spaces
}

\author{
Wei-Shih Du \\ Department of Mathematics, National Kaohsiung Normal University, Kaohsiung 802, Taiwan \\ Correspondence should be addressed to Wei-Shih Du,wsdu@nknucc.nknu.edu.tw \\ Received 10 November 2009; Revised 5 March 2010; Accepted 14 March 2010 \\ Academic Editor: Tomonari Suzuki
}

Copyright (C) 2010 Wei-Shih Du. This is an open access article distributed under the Creative Commons Attribution License, which permits unrestricted use, distribution, and reproduction in any medium, provided the original work is properly cited.

The main aim of this paper is to study and establish some new coupled fixed point theorems for nonlinear contractive maps that satisfied Mizoguchi-Takahashi's condition in the setting of quasiordered metric spaces or usual metric spaces.

\section{Introduction}

Let $(X, d)$ be a metric space. For each $x \in X$ and $A \subseteq X$, let $d(x, A)=\inf _{y \in A} d(x, y)$. Denote by $\mathcal{N}(X)$ the class of all nonempty subsets of $X$ and $C B(X)$ the family of all nonempty closed and bounded subsets of $X$. A function $\mathscr{d}: \mathcal{C} B(X) \times C B(X) \rightarrow[0, \infty)$ defined by

$$
\mathscr{H}(A, B)=\max \left\{\sup _{x \in B} d(x, A), \sup _{x \in A} d(x, B)\right\}
$$

is said to be the Hausdorff metric on $\mathcal{C} B(X)$ induced by the metric $d$ on $X$. A point $x$ in $X$ is a fixed point of a map $T$ if $T x=x$ (when $T: X \rightarrow X$ is a single-valued map) or $x \in T x$ (when $T: X \rightarrow \mathcal{N}(X)$ is a multivalued map). Throughout this paper we denote by $\mathbb{N}$ and $\mathbb{R}$ the set of positive integers and real numbers, respectively.

The existence of fixed point in partially ordered sets has been investigated recently in [1-11] and references therein. In [6, 8], Nieto and Rodríguez-López used Tarski's theorem to show the existence of solutions for fuzzy equations and fuzzy differential equations, respectively. The existence of solutions for matrix equations or ordinary differential equations 
by applying fixed point theorems is presented in $[2,4,7,9,10]$. The authors in $[3,11]$ proved some fixed point theorems for a mixed monotone mapping in a metric space endowed with partial order and applied their results to problems of existence and uniqueness of solutions for some boundary value problems.

The various contractive conditions are important to find the existence of fixed point. There is a trend to weaken the requirement on the contraction. In 1989, Mizoguchi and Takahashi [12] proved the following interesting fixed point theorem for a weak contraction which is a partial answer of Problem 9 in Reich [13] (see also [14-16] and references therein).

Theorem MT (Mizoguchi and Takahashi [12]). Let $(X, d)$ be a complete metric space and $T$ a map from $X$ into $C B(X)$. Assume that

$$
\mathscr{\ell}(T x, T y) \leq \varphi(d(x, y)) d(x, y)
$$

for all $x, y \in X$, where $\varphi$ is a function from $[0, \infty)$ into $[0,1)$ satisfying

$$
\limsup _{s \rightarrow t+0} \varphi(s)<1 \quad \forall t \in[0, \infty)
$$

Then there exists $v \in X$ such that $v \in T v$.

In fact, Mizoguchi-Takahashi's fixed point theorem is a generalization of Nadler's fixed point theorem $[17,18]$ which extended the Banach contraction principle (see, e.g., [18]) to multivalued maps, but its primitive proof is different. Recently, Suzuki [19] gave a very simple proof of Theorem MT.

The purpose of this paper is to present some new coupled fixed point theorems for weakly contractive maps that satisfied Mizoguchi-Takahashi's condition (i.e., $\limsup _{s \rightarrow t+0} \varphi(s)<1$ for all $\left.t \in[0, \infty)\right)$ in the setting of quasiordered metric spaces or usual metric spaces. Our results generalize and improve some results in $[2,7,9]$ and references therein.

\section{Generalized Bhaskar-Lakshmikantham's Coupled Fixed Point Theorems and Others}

Let $X$ be a nonempty set and " $\leq$ " a quasiorder (preorder or pseudo-order, i.e., a reflexive and transitive relation) on $X$. Then $(X, \preceq)$ is called a quasiordered set. A sequence $\left\{x_{n}\right\}_{n \in \mathbb{N}}$ is called $\preceq$-nondecreasing (resp., $\leq$-nonincreasing) if $x_{n} \preceq x_{n+1}$ (resp., $x_{n+1} \leq x_{n}$ ) for each $n \in \mathbb{N}$. Let $(X, d)$ be a metric space with a quasi-order $\leq((X, d, \preceq)$ for short $)$. We endow the product space $X \times X$ with the metric $\rho$ defined by

$$
\rho((x, y),(u, v))=d(x, u)+d(y, v) \text { for any }(x, y),(u, v) \in X \times X .
$$

A map $F: X \times X \rightarrow X$ is said to be continuous at $(\widehat{x}, \widehat{y}) \in X \times X$ if any sequence $\left\{\left(x_{n}, y_{n}\right)\right\} \subset$

$X \times X$ with $\left(x_{n}, y_{n}\right) \stackrel{\rho}{\rightarrow}(\widehat{x}, \widehat{y})$ implies $F\left(x_{n}, y_{n}\right) \stackrel{d}{\rightarrow} F(\widehat{x}, \widehat{y}) . F$ is said to be continuous on $X \times X$ if $F$ is continuous at every point of $X \times X$. 
In this paper, we also endow the product space $X \times X$ with the following quasi-order $\preccurlyeq:$

$$
(u, v) \preccurlyeq(x, y) \Longleftrightarrow u \preceq x \text { and } y \preceq v \quad \text { for any }(x, y),(u, v) \in X \times X .
$$

Definition 2.1 (see [2]). Let $(X, \preceq)$ be a quasiordered set and $F: X \times X \rightarrow X$ a map. We say that $F$ has the mixed monotone property on $X$ if $F(x, y)$ is monotone nondecreasing in $x \in X$ and is monotone nonincreasing in $x \in X$, that is, for any $x, y \in X$,

$$
\begin{aligned}
& x_{1}, x_{2} \in X \text { with } x_{1} \preceq x_{2} \Longrightarrow F\left(x_{1}, y\right) \preceq F\left(x_{2}, y\right) \\
& y_{1}, y_{2} \in X \text { with } y_{1} \preceq y_{2} \Longrightarrow F\left(x, y_{2}\right) \preceq F\left(x, y_{1}\right)
\end{aligned}
$$

It is quite obvious that if $F: X \times X \rightarrow X$ has the mixed monotone property on $X$, then for any $(x, y),(u, v) \in X \times X$ with $(u, v) \preccurlyeq(x, y)$ (i.e., $u \leq x$ and $y \leq v), F(u, v) \preceq F(x, y)$.

Definition 2.2 (see [2]). Let $X$ be a nonempty set and $F: X \times X \rightarrow X$ a map. We call an element $(x, y) \in X \times X$ a coupled fixed point of $F$ if

$$
F(x, y)=x, \quad F(y, x)=y \text {. }
$$

Definition 2.3. Let $(X, d)$ be a metric space with a quasi-order $\lesssim$. A nonempty subset $M$ of $X$ is said to be

(i) sequentially $\varsigma^{\uparrow}$-complete if every $\preceq$-nondecreasing Cauchy sequence in $M$ converges;

(ii) sequentially $\leq_{\downarrow}$-complete if every $\preceq$-nonincreasing Cauchy sequence in $M$ converges;

(iii) sequentially $\leq_{\downarrow}^{\uparrow}$-complete if it is both $\leq^{\uparrow}$-complete and $\leq_{\downarrow}$-complete.

Definition 2.4 (see [20]). A function $\varphi:[0, \infty) \rightarrow[0,1)$ is said to be a MT-function if it satisfies Mizoguchi-Takahashi's condition (i.e., $\limsup _{s \rightarrow t+0} \varphi(s)<1$ for all $t \in[0, \infty)$ ).

Remark 2.5 .

(i) Obviously, if $\varphi:[0, \infty) \rightarrow[0,1)$ is defined by $\varphi(t)=c$, where $c \in[0,1)$, then $\varphi$ is a $M T$-function.

(ii) If $\varphi:[0, \infty) \rightarrow[0,1)$ is a nondecreasing function, then $\varphi$ is a MT-function.

(iii) Notice that $\varphi:[0, \infty) \rightarrow[0,1)$ is a MT-function if and only if for each $t \in[0, \infty)$ there exist $r_{t} \in[0,1)$ and $\varepsilon_{t}>0$ such that $\varphi(s) \leq r_{t}$ for all $s \in\left[t, t+\varepsilon_{t}\right)$. Indeed, if $\varphi$ is a $M T$-function, then $\lim \sup _{s \rightarrow t+0} \varphi(s)<1$ for all $t \in[0, \infty)$. So for each $t \in[0, \infty)$ there exists $\varepsilon_{t}>0$ such that $\sup _{s \in\left[t, t+\varepsilon_{t}\right)} \varphi(s)<1$. Therefore we can find $r_{t} \in[0,1)$ such that $\sup _{s \in\left[t, t+\varepsilon_{t}\right)} \varphi(s) \leq r_{t}<1$, and hence $\varphi(s) \leq r_{t}$ for all $s \in\left[t, t+\varepsilon_{t}\right)$. The converse part is obvious.

The following lemmas are crucial to our proofs.

Lemma 2.6 (see $[20])$. Let $\varphi:[0, \infty) \rightarrow[0,1)$ be a MT-function. Then $\kappa:[0, \infty) \rightarrow[0,1)$ defined by $\kappa(t)=(\varphi(t)+1) / 2$ is also a MT-function. 
Proof. Clearly, $\kappa(t)>\varphi(t)$ and $0<\kappa(t)<1$ for all $t \in[0, \infty)$. Let $t \in[0, \infty)$ be fixed. Since $\varphi:[0, \infty) \rightarrow[0,1)$ is a MT-function, there exist $r_{t} \in[0,1)$ and $\varepsilon_{t}>0$ such that $\varphi(s) \leq r_{t}$ for all $s \in\left[t, t+\varepsilon_{t}\right)$. Let $\lambda_{t}:=\left(r_{t}+1\right) / 2 \in[0,1)$. Then $\kappa(s) \leq \lambda_{t}$ for all $s \in\left[t, t+\varepsilon_{t}\right)$ and hence $\kappa$ is a $M T$-function.

Lemma 2.7. Let $(X, \leq)$ be a quasiordered set and $F: X \times X \rightarrow X$ a map having the mixed monotone property on $X$. Let $x_{0}, y_{0} \in X$. Define two sequences $\left\{x_{n}\right\}$ and $\left\{y_{n}\right\}$ by

$$
\begin{aligned}
& x_{n}=F\left(x_{n-1}, y_{n-1}\right), \\
& y_{n}=F\left(y_{n-1}, x_{n-1}\right)
\end{aligned}
$$

for each $n \in \mathbb{N}$. If $x_{0} \preceq x_{1}$ and $y_{1} \preceq y_{0}$, then $\left\{x_{n}\right\}$ is $\preceq$-nondecreasing and $\left\{y_{n}\right\}$ is $\preceq$-nonincreasing.

Proof. Since $x_{0} \preceq x_{1}$ and $y_{1} \preceq y_{0}$, by (2.5), and the mixed monotone property of $F$, we have

$$
\begin{aligned}
& x_{1}=F\left(x_{0}, y_{0}\right) \leq F\left(x_{0}, y_{1}\right) \leq F\left(x_{1}, y_{1}\right)=x_{2}, \\
& y_{2}=F\left(y_{1}, x_{1}\right) \leq F\left(y_{0}, x_{1}\right) \leq F\left(y_{0}, x_{0}\right)=y_{1} .
\end{aligned}
$$

Let $k \in \mathbb{N}$ and assume that $x_{k-1} \preceq x_{k}$ and $y_{k} \leq y_{k-1}$ is already known. Then

$$
\begin{aligned}
& x_{k}=F\left(x_{k-1}, y_{k-1}\right) \leq F\left(x_{k-1}, y_{k}\right) \leq F\left(x_{k}, y_{k}\right)=x_{k+1}, \\
& y_{k+1}=F\left(y_{k}, x_{k}\right) \leq F\left(y_{k-1}, x_{k}\right) \leq F\left(y_{k-1}, x_{k-1}\right)=y_{k} .
\end{aligned}
$$

Hence, by induction, we prove that $\left\{x_{n}\right\}$ is $\preceq$-nondecreasing and $\left\{y_{n}\right\}$ is $\preceq$-nonincreasing.

Theorem 2.8. Let $(X, d, \preceq)$ be a sequentially $\leq_{\downarrow}^{\uparrow}$-complete metric space and $F: X \times X \rightarrow X a$ continuous map having the mixed monotone property on $X$. Assume that there exists a MT-function $\varphi:[0, \infty) \rightarrow[0,1)$ such that for any $(x, y),(u, v) \in X \times X$ with $(u, v) \preccurlyeq(x, y)$,

$$
d(F(x, y), F(u, v)) \leq \frac{1}{2} \varphi(\rho((x, y),(u, v))) \rho((x, y),(u, v)) .
$$

If there exist $x_{0}, y_{0} \in X$ such that $x_{0} \leq F\left(x_{0}, y_{0}\right)$ and $F\left(y_{0}, x_{0}\right) \leq y_{0}$, then there exist $\widehat{x}, \hat{y} \in X$, such that $\widehat{x}=F(\widehat{x}, \widehat{y})$ and $\widehat{y}=F(\widehat{y}, \widehat{x})$.

Proof. By Lemma 2.6, we can define a MT-function $\mathcal{\kappa}:[0, \infty) \rightarrow[0,1)$ by $\kappa(t)=(\varphi(t)+1) / 2$. Then $\varphi(t)<\kappa(t)$ and $0<\kappa(t)<1$ for all $t \in[0, \infty)$. For any $n \in \mathbb{N}$, let $x_{n}=F\left(x_{n-1}, y_{n-1}\right)$ and $y_{n}=F\left(y_{n-1}, x_{n-1}\right)$ be defined as in Lemma 2.7. Then, by Lemma 2.7, $\left\{x_{n}\right\}$ is $\preceq$-nondecreasing 
and $\left\{y_{n}\right\}$ is $\preceq$-nonincreasing. So $\left(x_{n}, y_{n}\right) \preccurlyeq\left(x_{n+1}, y_{n+1}\right)$ and $\left(y_{n+1}, x_{n+1}\right) \preccurlyeq\left(y_{n}, x_{n}\right)$ for each $n \in \mathbb{N}$. By (2.8), we obtain

$$
\begin{aligned}
d\left(x_{2}, x_{1}\right) & =d\left(F\left(x_{1}, y_{1}\right), F\left(x_{0}, y_{0}\right)\right) \\
& <\frac{1}{2} \kappa\left(\rho\left(\left(x_{1}, y_{1}\right),\left(x_{0}, y_{0}\right)\right)\right) \rho\left(\left(x_{1}, y_{1}\right),\left(x_{0}, y_{0}\right)\right) \\
& =\frac{1}{2} \kappa\left(d\left(x_{1}, x_{0}\right)+d\left(y_{1}, y_{0}\right)\right)\left[d\left(x_{1}, x_{0}\right)+d\left(y_{1}, y_{0}\right)\right] \\
d\left(y_{2}, y_{1}\right) & =d\left(y_{1}, y_{2}\right) \\
& =d\left(F\left(y_{0}, x_{0}\right), F\left(y_{1}, x_{1}\right)\right) \\
& <\frac{1}{2} \kappa\left(d\left(y_{0}, y_{1}\right)+d\left(x_{0}, x_{1}\right)\right)\left[d\left(y_{0}, y_{1}\right)+d\left(x_{0}, x_{1}\right)\right] \\
& =\frac{1}{2} \kappa\left(d\left(x_{1}, x_{0}\right)+d\left(y_{1}, y_{0}\right)\right)\left[d\left(x_{1}, x_{0}\right)+d\left(y_{1}, y_{0}\right)\right]
\end{aligned}
$$

It follows that

$$
d\left(x_{2}, x_{1}\right)+d\left(y_{2}, y_{1}\right)<\kappa\left(d\left(x_{1}, x_{0}\right)+d\left(y_{1}, y_{0}\right)\right)\left[d\left(x_{1}, x_{0}\right)+d\left(y_{1}, y_{0}\right)\right]
$$

For each $n \in \mathbb{N}$, let $\xi_{n}=d\left(x_{n}, x_{n-1}\right)+d\left(y_{n}, y_{n-1}\right)$. Then $\xi_{2}<\kappa\left(\xi_{1}\right) \xi_{1}$. By induction, we can obtain the following: for each $n \in \mathbb{N}$,

$$
\begin{aligned}
d\left(x_{n+1}, x_{n}\right) & <\frac{1}{2} \kappa\left(\xi_{n}\right) \xi_{n} \\
d\left(y_{n+1}, y_{n}\right) & <\frac{1}{2} \kappa\left(\xi_{n}\right) \xi_{n} \\
\xi_{n+1} & <\kappa\left(\xi_{n}\right) \xi_{n} .
\end{aligned}
$$

Since $0<\kappa(t)<1$ for all $t \in[0, \infty)$, the sequence $\left\{\xi_{n}\right\}$ is strictly decreasing in $[0, \infty)$ from (2.13). Let $\delta:=\lim _{n \rightarrow \infty} \xi_{n}=\inf _{n \in \mathbb{N}} \xi_{n} \geq 0$. Since $\mathcal{\kappa}$ is a $M T$-function, there exists $\gamma \in(0,1)$ and $\varepsilon>0$ such that $\kappa(s) \leq \gamma$ for all $s \in[\delta, \delta+\varepsilon)$. Also, there exists $\ell \in \mathbb{N}$ such that

$$
\delta \leq \xi_{n}<\delta+\varepsilon
$$


for all $n \in \mathbb{N}$ with $n \geq \ell$. So $\kappa\left(\xi_{n+\ell-1}\right) \leq \gamma$ for each $n \in \mathbb{N}$. Let $a_{n}=x_{n+\ell-1}$ and $b_{n}=y_{n+\ell-1}, n \in \mathbb{N}$. We claim that $\left\{a_{n}\right\}$ is a $\preceq$-nondecreasing Cauchy sequence in $X$ and $\left\{b_{n}\right\}$ is a $\preceq$-nonincreasing Cauchy sequence in $X$. Indeed, from our hypothesis, for each $n \in \mathbb{N}$, we have

$$
\begin{aligned}
d\left(a_{n+2}, a_{n+1}\right) & =d\left(x_{n+\ell+1}, x_{n+\ell}\right) \\
& <\frac{1}{2} \kappa\left(\xi_{n+\ell}\right) \xi_{n+\ell} \quad(\operatorname{by}(2.11)) \\
& \leq \frac{1}{2} \gamma\left[d\left(x_{n+\ell}, x_{n+\ell-1}\right)+d\left(y_{n+\ell}, y_{n+\ell-1}\right)\right] \\
& =\frac{1}{2} \gamma\left[d\left(a_{n+1}, a_{n}\right)+d\left(b_{n+1}, b_{n}\right)\right] .
\end{aligned}
$$

Similarly,

$$
d\left(b_{n+2}, b_{n+1}\right)<\frac{1}{2} \gamma\left[d\left(a_{n+1}, a_{n}\right)+d\left(b_{n+1}, b_{n}\right)\right] .
$$

Hence we get

$$
d\left(a_{n+2}, a_{n+1}\right)+d\left(b_{n+2}, b_{n+1}\right)<\gamma\left[d\left(a_{n+1}, a_{n}\right)+d\left(b_{n+1}, b_{n}\right)\right] \text { for each } n \in \mathbb{N} \text {. }
$$

So it follows from (2.17) that

$$
\begin{aligned}
d\left(a_{n+2}, a_{n+1}\right) & <\frac{1}{2} \gamma\left[d\left(a_{n+1}, a_{n}\right)+d\left(b_{n+1}, b_{n}\right)\right] \\
& <\frac{1}{2} \gamma^{2}\left[d\left(a_{n}, a_{n-1}\right)+d\left(b_{n}, b_{n-1}\right)\right] \\
& <\cdots \\
& <\frac{1}{2} \gamma^{n}\left[d\left(a_{2}, a_{1}\right)+d\left(b_{2}, b_{1}\right)\right], \\
d\left(b_{n+2}, b_{n+1}\right) & <\frac{1}{2} \gamma^{n}\left[d\left(a_{2}, a_{1}\right)+d\left(b_{2}, b_{1}\right)\right] \quad \text { for } n \in \mathbb{N} .
\end{aligned}
$$

Let $\lambda_{n}=\left(\gamma^{n-1} / 2(1-\gamma)\right)\left[d\left(a_{2}, a_{1}\right)+d\left(b_{2}, b_{1}\right)\right], n \in \mathbb{N}$. For $m, n \in \mathbb{N}$ with $m>n$, we have

$$
\begin{aligned}
& d\left(a_{m}, a_{n}\right) \leq \sum_{j=n}^{m-1} d\left(a_{j+1}, a_{j}\right)<\lambda_{n} \\
& d\left(b_{m}, b_{n}\right) \leq \sum_{j=n}^{m-1} d\left(b_{j+1}, b_{j}\right)<\lambda_{n} .
\end{aligned}
$$


Since $0<\gamma<1, \lim _{n \rightarrow \infty} \lambda_{n}=0$ and hence

$$
\lim _{n \rightarrow \infty} \sup \left\{d\left(a_{m}, a_{n}\right): m>n\right\}=\lim _{n \rightarrow \infty} \sup \left\{d\left(b_{m}, b_{n}\right): m>n\right\}=0
$$

So $\left\{a_{n}\right\}$ is a $\preceq$-nondecreasing Cauchy sequence in $X$ and $\left\{b_{n}\right\}$ is a $\preceq$-nonincreasing Cauchy sequence in $X$. By the sequentially $\leq_{\downarrow}^{\uparrow}$-completeness of $X$, there exist $\widehat{x}, \hat{y} \in X$ such that $a_{n} \rightarrow$ $\widehat{x}$ and $b_{n} \rightarrow \widehat{y}$ as $n \rightarrow \infty$. Hence $x_{n} \rightarrow \widehat{x}$ and $y_{n} \rightarrow \widehat{y}$ as $n \rightarrow \infty$.

Let $\varepsilon>0$ be given. Since $F$ is continuous at $(\widehat{x}, \widehat{y})$, there exists $\delta>0$ such that

$$
d(F(\widehat{x}, \widehat{y}), F(u, v))<\frac{\varepsilon}{2}
$$

whenever $(u, v) \in X \times X$ with $\rho((\widehat{x}, \widehat{y}),(u, v))<\delta$. Since $x_{n} \rightarrow \widehat{x}$ and $y_{n} \rightarrow \widehat{y}$ as $n \rightarrow \infty$, for $\zeta=\min \{\varepsilon / 2, \delta / 2\}>0$, there exist $n_{0} \in \mathbb{N}$ such that

$$
d\left(x_{n}, \widehat{x}\right)<\zeta, \quad d\left(y_{n}, \widehat{y}\right)<\zeta \quad \forall n \in \mathbb{N} \text { with } n \geq n_{0} .
$$

So, for each $n \in \mathbb{N}$ with $n \geq n_{0}$, by (2.22),

$$
\rho\left((\widehat{x}, \widehat{y}),\left(x_{n}, y_{n}\right)\right)=d\left(x_{n}, \widehat{x}\right)+d\left(y_{n}, \widehat{y}\right)<\delta,
$$

and hence we have from (2.21) that

$$
d\left(F(\widehat{x}, \widehat{y}), F\left(x_{n}, y_{n}\right)\right)<\frac{\varepsilon}{2}
$$

Therefore

$$
\begin{aligned}
d(F(\widehat{x}, \widehat{y}), \widehat{x}) & \leq d\left(F(\widehat{x}, \widehat{y}), x_{n_{0}+1}\right)+d\left(x_{n_{0}+1}, \widehat{x}\right) \\
& =d\left(F(\widehat{x}, \widehat{y}), F\left(x_{n_{0}}, y_{n_{0}}\right)\right)+d\left(x_{n_{0}+1}, \widehat{x}\right) \\
& <\frac{\varepsilon}{2}+\zeta \quad(\text { by }(2.22) \text { and }(2.24)) \\
& \leq \varepsilon .
\end{aligned}
$$

Since $\varepsilon$ is arbitrary, $d(F(\widehat{x}, \widehat{y}), \widehat{x})=0$ or $F(\widehat{x}, \widehat{y})=\widehat{x}$. Similarly, we can also prove that $F(\widehat{y}, \widehat{x})=$ $\widehat{y}$. The proof is completed.

Remark 2.9. Theorem 2.8 generalizes and improves Bhaskar-Lakshmikantham's coupled fixed points theorem [2, Theorem 2.1] and some results in [7, 9].

Following a similar argument as in the proof of [2, Theorem 2.2] and applying Theorem 2.8 , one can verify the following result where $F$ is not necessarily continuous. 
Theorem 2.10. Let $(X, d, \preceq)$ be a sequentially $\leq_{\downarrow}^{\uparrow}$-complete metric space and $F: X \times X \rightarrow X$ a map having the mixed monotone property on X. Assume that

(i) any $\leq$-nondecreasing sequence $\left\{x_{n}\right\}$ with $x_{n} \rightarrow \hat{x}$ implies $x_{n} \preceq \hat{x}$ for each $n \in \mathbb{N}$;

(ii) any $\leq$-nonincreasing sequence $\left\{y_{n}\right\}$ with $y_{n} \rightarrow \widehat{y}$ implies $\widehat{y} \preceq y_{n}$ for each $n \in \mathbb{N}$;

(iii) there exists a MT-function $\varphi:[0, \infty) \rightarrow[0,1)$ such that for any $(x, y),(u, v) \in X \times X$ with $(u, v) \preccurlyeq(x, y)$,

$$
d(F(x, y), F(u, v)) \leq \frac{1}{2} \varphi(\rho((x, y),(u, v))) \rho((x, y),(u, v)) .
$$

If there exist $x_{0}, y_{0} \in X$ such that $x_{0} \leq F\left(x_{0}, y_{0}\right)$ and $F\left(y_{0}, x_{0}\right) \preceq y_{0}$, then there exist $\hat{x}$, $\widehat{y} \in X$, such that $\widehat{x}=F(\widehat{x}, \widehat{y})$ and $\widehat{y}=F(\widehat{y}, \widehat{x})$.

Remark 2.11.

(a) [2, Theorem 2.2] is a special case of Theorem 2.10.

(b) Similarly, we can obtain the generalizations of Theorems 2.4-2.6 in [2] for MTfunctions.

Finally, we discuss the following coupled fixed point theorem in (usual) complete metric spaces.

Theorem 2.12. Let $(X, d)$ be a complete metric space and $F: X \times X \rightarrow X$ a map. Assume that there exists a MT-function $\varphi:[0, \infty) \rightarrow[0,1)$ such that for any $(x, y),(u, v) \in X \times X$,

$$
d(F(x, y), F(u, v)) \leq \frac{1}{2} \varphi(\rho((x, y),(u, v))) \rho((x, y),(u, v)) .
$$

Then $F$ has a unique coupled fixed point in $X \times X$; that is, there exists unique $(\widehat{x}, \hat{y}) \in X \times X$, such that $\hat{x}=F(\widehat{x}, \hat{y})$ and $\hat{y}=F(\widehat{y}, \widehat{x})$.

Proof. Let $x_{0}, y_{0} \in X$ be given. For any $n \in \mathbb{N}$, define $x_{n}=F\left(x_{n-1}, y_{n-1}\right)$ and $y_{n}=F\left(y_{n-1}, x_{n-1}\right)$. By our hypothesis, we know that $F$ is continuous. Following the same argument as in the proof of Theorem 2.8, there exists $(\widehat{x}, \widehat{y}) \in X \times X$, such that $\widehat{x}=F(\widehat{x}, \widehat{y})$ and $\widehat{y}=F(\widehat{y}, \widehat{x})$. We prove the uniqueness of the coupled fixed point of $F$. On the contrary, suppose that there exists $(\widehat{u}, \widehat{v}) \in X \times X$, such that $\widehat{u}=F(\widehat{u}, \widehat{v})$ and $\widehat{v}=F(\widehat{v}, \widehat{u})$. Then we obtain

$$
\begin{aligned}
& d(\widehat{x}, \widehat{u})=d(F(\widehat{x}, \widehat{y}), F(\widehat{u}, \widehat{v}))<\frac{1}{2}[d(\widehat{x}, \widehat{u})+d(\widehat{y}, \widehat{v})], \\
& d(\widehat{y}, \widehat{v})=d(F(\widehat{y}, \widehat{x}), F(\widehat{v}, \widehat{u}))<\frac{1}{2}[d(\widehat{x}, \widehat{u})+d(\widehat{y}, \widehat{v})] .
\end{aligned}
$$

It follows from (2.28) that

$$
d(\widehat{x}, \widehat{u})+d(\widehat{y}, \widehat{v})<d(\widehat{x}, \widehat{u})+d(\widehat{y}, \widehat{v})
$$

a contradiction. The proof is completed. 


\section{Acknowledgment}

This paper is dedicated to Professor Wataru Takahashi in celebration of his retirement. This research was supported by the National Science Council of the Republic of China.

\section{References}

[1] R. P. Agarwal, M. A. El-Gebeily, and D. O’Regan, “Generalized contractions in partially ordered metric spaces," Applicable Analysis, vol. 87, no. 1, pp. 109-116, 2008.

[2] T. G. Bhaskar and V. Lakshmikantham, "Fixed point theorems in partially ordered metric spaces and applications," Nonlinear Analysis: Theory, Methods E Applications, vol. 65, no. 7, pp. 1379-1393, 2006.

[3] D. Burgić, S. Kalabušić, and M. R. S. Kulenović, "Global attractivity results for mixed-monotone mappings in partially ordered complete metric spaces," Fixed Point Theory and Applications, vol. 2009, Article ID 762478, 17 pages, 2009.

[4] J. Harjani and K. Sadarangani, "Fixed point theorems for weakly contractive mappings in partially ordered sets," Nonlinear Analysis: Theory, Methods \& Applications, vol. 71, no. 7-8, pp. 3403-3410, 2009.

[5] V. Lakshmikantham and L. Cirić, "Coupled fixed point theorems for nonlinear contractions in partially ordered metric spaces," Nonlinear Analysis: Theory, Methods \& Applications, vol. 70, no. 12, pp. 4341-4349, 2009.

[6] J. J. Nieto and R. Rodríguez-López, "Existence of extremal solutions for quadratic fuzzy equations," Fixed Point Theory and Applications, vol. 2005, no. 3, pp. 321-342, 2005.

[7] J. J. Nieto and R. Rodríguez-López, "Contractive mapping theorems in partially ordered sets and applications to ordinary differential equations," Order, vol. 22, no. 3, pp. 223-239, 2005.

[8] J. J. Nieto and R. Rodríguez-López, "Applications of contractive-like mapping principles to fuzzy equations," Revista Matemática Complutense, vol. 19, no. 2, pp. 361-383, 2006.

[9] J. J. Nieto and R. Rodríguez-López, "Existence and uniqueness of fixed point in partially ordered sets and applications to ordinary differential equations," Acta Mathematica Sinica, vol. 23, no. 12, pp. 2205-2212, 2007.

[10] A. C. M. Ran and M. C. B. Reurings, "A fixed point theorem in partially ordered sets and some applications to matrix equations," Proceedings of the American Mathematical Society, vol. 132, no. 5, pp. 1435-1443, 2004.

[11] Y. Wu, "New fixed point theorems and applications of mixed monotone operator," Journal of Mathematical Analysis and Applications, vol. 341, no. 2, pp. 883-893, 2008.

[12] N. Mizoguchi and W. Takahashi, "Fixed point theorems for multivalued mappings on complete metric spaces," Journal of Mathematical Analysis and Applications, vol. 141, no. 1, pp. 177-188, 1989.

[13] S. Reich, "Some problems and results in fixed point theory," in Topological Methods in Nonlinear Functional Analysis (Toronto, Ont., 1982), vol. 21 of Contemp. Math., pp. 179-187, American Mathematical Society, Providence, RI, USA, 1983.

[14] P. Z. Daffer, H. Kaneko, and W. Li, "On a conjecture of S. Reich," Proceedings of the American Mathematical Society, vol. 124, no. 10, pp. 3159-3162, 1996.

[15] J. Jachymski, “On Reich's question concerning fixed points of multimaps," Bollettino dell'Unione Matematica Italiana, vol. 9, no. 3, pp. 453-460, 1995.

[16] P. V. Semenov, "Fixed points of multivalued contractions," Functional Analysis and Its Applications, vol. 36, no. 2, pp. 159-161, 2002.

[17] S. B. Nadler Jr., "Multi-valued contraction mappings," Pacific Journal of Mathematics, vol. 30, pp. 475488, 1969.

[18] W. Takahashi, Nonlinear Functional Analysis, Fixed Point Theory and Its Applications, Yokohama, Yokohama, Japan, 2000.

[19] T. Suzuki, "Mizoguchi-Takahashi's fixed point theorem is a real generalization of Nadler's," Journal of Mathematical Analysis and Applications, vol. 340, no. 1, pp. 752-755, 2008.

[20] W.-S. Du, "Some new results and generalizations in metric fixed point theory," Nonlinear Analysis: Theory, Methods and Applications (2010). 